\title{
Low lean mass is associated with lower urinary tract symptoms in US men from the 2005-2006 national health and nutrition examination survey dataset
}

\author{
Zheng Qin ${ }^{1,2,3}$, Junjie Zhao4, Jiameng $\mathrm{Li}^{1,2,3}$, Qinbo Yang ${ }^{1,2,3}$, Jiwen Geng ${ }^{1,2,3}$, Ruoxi Liao ${ }^{1,2,3}$, \\ Baihai Su ${ }^{1,2,3}$ \\ ${ }^{1}$ Department of Nephrology, National Clinical Research Center for Geriatrics, West China Hospital of Sichuan \\ University, Chengdu 610041, China \\ ${ }^{2}$ Med+ Biomaterial Institute of West China Hospital/West China School of Medicine of Sichuan University, \\ Chengdu 610041, China \\ ${ }^{3}$ Med-X Center for Materials, Sichuan University, Chengdu 610041, China \\ ${ }^{4}$ West China School of Medicine, West China Hospital of Sichuan University, Chengdu 610041, China
}

Correspondence to: Baihai Su; email: imsbh@163.com, https://orcid.org/0000-0002-2187-8168

Keywords: low lean mass, lower urinary tract symptoms, NHANES, cross-sectional study

Received: April 27, 2021

Accepted: August 14, 2021

Published: September 2, 2021

Copyright: (C) 2021 Qin et al. This is an open access article distributed under the terms of the Creative Commons Attribution License (CC BY 3.0), which permits unrestricted use, distribution, and reproduction in any medium, provided the original author and source are credited.

\section{ABSTRACT}

We investigated the relationship between low lean mass (LLM) and lower urinary tract symptoms (LUTS) using the 2005-2006 National Health and Nutrition Examination Survey (NHANES) dataset. We enrolled 959 men with an average age of $\mathbf{5 2 . 0 8} \pm \mathbf{7 . 9 1}$ years and performed weighted multiple regression analysis to determine the independent relationship between exposure variables (LLM, alternate LLM) and outcomes variables (urinary hesitancy, incomplete emptying, urinary frequency, nocturia, daytime LUTS, clinical LUTS) after adjusting for confounding factors. The prevalence of urinary hesitancy $(O R=7.76, P<0.0001)$, incomplete emptying $(O R=$ 2.49, $P=0.0070)$, urinary frequency $(O R=3.28, P<0.0001)$, daytime LUTS $(O R=3.88, P<0.0001)$ and clinical LUTS $(O R=8.11, P<0.0001)$ was significantly higher among men with LLM compared to men without LLM. Moreover, alternate LLM (ALLM) was positively associated with urinary hesitancy $(O R=17.97, P<0.0001)$, incomplete emptying (OR = 4.68, $P=0.0003)$, daytime $L U T S(O R=2.47, P=0.0136)$ and clinical $L U T S(O R=12.18, P<0.0001)$. These findings demonstrate that both LLM and ALLM were associated with a higher risk of LUTS in men aged $\geq$ 40 years, which suggested that early management and treatment of lean mass loss may improve or alleviate LUTS.

\section{INTRODUCTION}

Lower urinary tract symptoms (LUTS) such as urinary hesitancy, incomplete emptying, urinary frequency and nocturia remained the most common urologic symptoms that significantly affect the quality of life of more than $90 \%$ of men aged $50 P<80$ years [1-4]. In the United States, the annual expenditure for LUTS medications is nearly 194 million dollars and represents a significant economic and public health burden [5, 6]. Therefore, there is an urgent need to identify risk factors associated with LUTS.

Low lean mass (LLM) is a key clinical indicator of muscle mass, strength and function [7]. The Foundation for the National Institutes of Health (FNIH) Sarcopenia 
project data demonstrated that LLM could be used to define sarcopenia, a skeletal muscle disorder that involves age-dependent decline in muscle mass and functional capacity [8]. Several other studies have also shown that LLM could serve as a underlying risk factor for muscle weakness, disability and mortality in the elderly population [9-12].

Although both LLM and LUTS are age-related diseases, the association between them still remains unclear and the knowledge about this topic is limited. Aging induces several changes in body composition, including increased visceral fat and decreased muscle mass. Obesity in the elderly with benign prostatic enlargement could increase the risk of LUTS [13]. Moreover, LLM has been reported to be associated with obesity [14]. Besides, previous studies on community-dwelling adults and older men showed a positive correlation between frailty and LUTS [15, 16]. Huang et al. showed that urinary incontinence was an independent risk factor associated with frailty [17]. Fougere et al. also reported that LLM was partly associated with frailty [18], it can be speculated that there might be a relationship between LLM and LUTS.

Hence, in this study, we explore the association between LLM and LUTS using the 2005-2006 National Health and Nutrition Examination Survey (NHANES) data regarding US men in order to provide more information about LLM and LUTS, which may shed new light on the management and intervention of LUTS at a younger age in clinical practice. We hypothesized that men with LLM were at an increased risk for LUTS.

\section{RESULTS}

\section{Baseline characteristics of NHANES study participants}

The average age of the 959 men included in our study was $52.08 \pm 7.91$ years. LLM and alternate LLM (ALLM) was reported by $143(14.91 \%)$ and $55(5.74 \%)$ participants, respectively. Urinary hesitancy, incomplete urine emptying, urinary frequency, and nocturia was reported by 68 (7.10\%), 97 (10.11\%), 141 (14.70\%), and 266 (27.74\%) participants, respectively. Daytime LUTS and clinical LUTS (having two or more symptoms above) was reported in $26.38 \%(n=253)$ and $13.97 \%(n=134)$ of the study participants, respectively (Table 1, Figure 1).

\section{Association between LLM and other clinicopathological characteristics of NHANES study subjects}

Table 1 shows details of weighted demographic characteristics and other covariates of the included

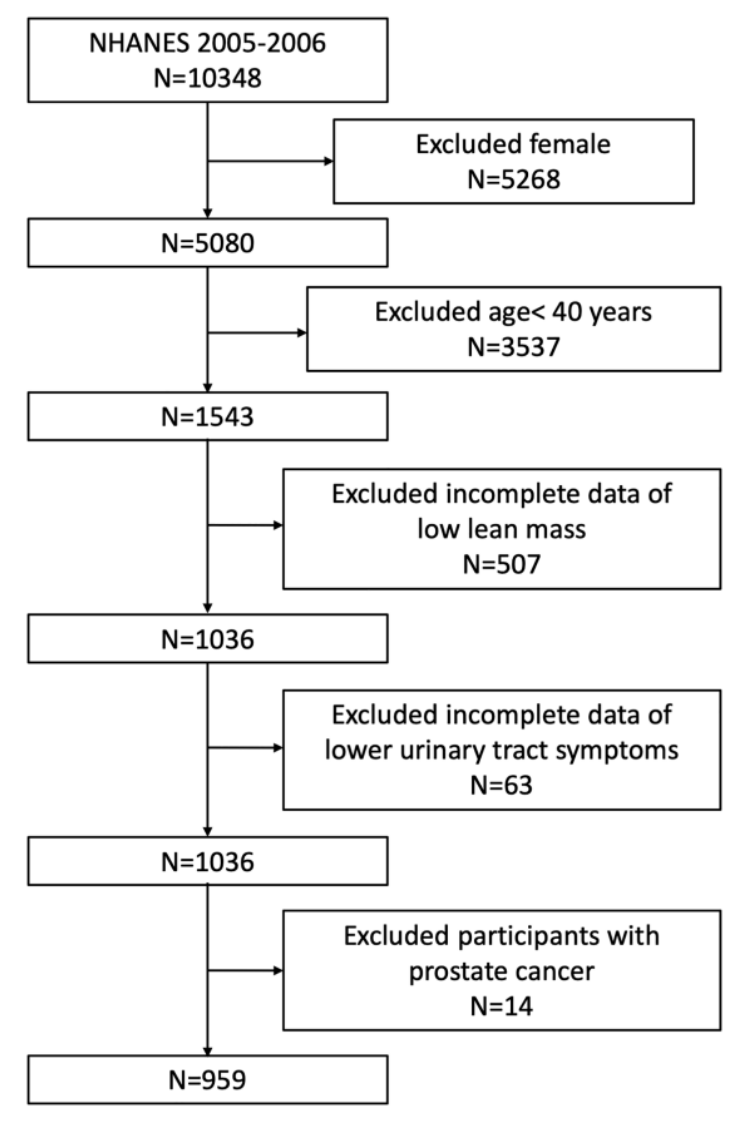

Figure 1. Flowchart of the sample selection from NHANES 2005-2006. 
Table 1. Baseline characteristics of participants, weighted.

\begin{tabular}{|c|c|c|c|c|}
\hline & Overall & Low lean mass & Non-low lean mass & \multirow{2}{*}{$P$ value } \\
\hline & $(n=959)$ & $(n=143)$ & $(n=816)$ & \\
\hline Age (years) & $52.08 \pm 7.91$ & $54.51 \pm 8.87$ & $51.72 \pm 7.70$ & 0.0003 \\
\hline \multicolumn{5}{|l|}{ Race $(\%)$} \\
\hline Mexican American & 6.30 & 11.77 & 5.51 & \multirow[t]{5}{*}{0.0001} \\
\hline Other Hispanic & 2.43 & 6.65 & 1.81 & \\
\hline Non-Hispanic White & 77.08 & 75.10 & 77.37 & \\
\hline Non-Hispanic Black & 9.98 & 4.30 & 10.81 & \\
\hline Other Races & 4.21 & 2.18 & 4.50 & \\
\hline \multicolumn{5}{|l|}{ Education level (\%) } \\
\hline Less than high school & 5.82 & 12.11 & 4.91 & \multirow[t]{3}{*}{0.0043} \\
\hline High school or GED & 35.91 & 37.16 & 35.73 & \\
\hline Above high school & 58.27 & 50.73 & 59.37 & \\
\hline \multicolumn{5}{|l|}{$\operatorname{RIP}(\%)$} \\
\hline$\leq 1$ & 8.30 & 10.21 & 8.29 & \multirow[t]{2}{*}{0.4840} \\
\hline$>1$ & 91.70 & 89.79 & 91.71 & \\
\hline \multicolumn{5}{|l|}{ Alcohol intake per week (\%) } \\
\hline Never & 20.19 & 24.17 & 19.62 & \multirow[t]{5}{*}{0.6712} \\
\hline Up to once a week & 41.44 & 42.55 & 41.28 & \\
\hline $2-3$ times a week & 16.77 & 15.87 & 16.90 & \\
\hline 4-6 times a week & 11.45 & 8.44 & 11.88 & \\
\hline Daily or more & 10.15 & 8.97 & 10.32 & \\
\hline \multicolumn{5}{|l|}{ BMI (\%) } \\
\hline Normal weight & 20.66 & 6.13 & 22.78 & \multirow[t]{3}{*}{$<0.0001$} \\
\hline Overweight & 40.28 & 25.32 & 42.46 & \\
\hline Obese & 39.06 & 68.54 & 34.77 & \\
\hline Insurance $(\%)$ & 83.45 & 71.89 & 85.13 & 0.0002 \\
\hline Hypertension (\%) & 38.61 & 7.30 & 43.15 & $<0.0001$ \\
\hline Diabetes $(\%)$ & 12.73 & 3.48 & 14.08 & 0.0011 \\
\hline Congestive heart failure $(\%)$ & 4.69 & 0.55 & 5.29 & 0.0211 \\
\hline COPD $(\%)$ & 4.77 & 0.56 & 4.93 & 0.5505 \\
\hline Coronary artery disease $(\%)$ & 5.43 & 3.69 & 6.14 & 0.0112 \\
\hline Cancer $(\%)$ & 3.63 & 2.45 & 3.80 & 0.4584 \\
\hline Sleep disorder $(\%)$ & 9.74 & 5.49 & 10.35 & 0.2012 \\
\hline \multicolumn{5}{|l|}{ Smoking status (\%) } \\
\hline Never & 39.71 & 1.88 & 45.19 & \multirow[t]{3}{*}{$<0.0001$} \\
\hline Former & 33.64 & 9.74 & 37.11 & \\
\hline Current & 26.65 & 88.38 & 17.70 & \\
\hline \multicolumn{5}{|l|}{ Comorbidity index (\%) } \\
\hline 0 & 50.96 & 86.291 & 45.83 & \multirow[t]{4}{*}{$<0.0001$} \\
\hline 1 & 34.60 & 9.99 & 38.17 & \\
\hline 2 & 9.98 & 3.11 & 10.97 & \\
\hline$\geq 3$ & 4.47 & 0.61 & 5.03 & \\
\hline Urinary hesitancy $(\%)$ & 7.26 & 16.50 & 5.92 & $<0.0001$ \\
\hline Incomplete emptying (\%) & 10.28 & 14.75 & 9.63 & 0.0827 \\
\hline Urinary frequency $(\%)$ & 13.52 & 18.37 & 12.82 & 0.0941 \\
\hline Nocturia $(\%)$ & 25.33 & 28.20 & 24.92 & 0.4377 \\
\hline Daytime LUTS (\%) & 25.53 & 35.44 & 24.09 & 0.0073 \\
\hline Clinical LUTS (\%) & 13.11 & 27.66 & 11.00 & $<0.0001$ \\
\hline
\end{tabular}

Abbreviations: BMI: body mass index; GED: general educational development; RIP: ratio of family income to poverty; COPD: chronic obstructive pulmonary disease; LUTS: lower urinary tract symptoms. 
participants with or without LLM. We observed significantly higher prevalence of older $(54.51 \pm 8.87 \mathrm{vs}$. $51.72 \pm 7.70$ years, $P=0.0003)$, obese $(68.54 \%$ vs. $34.77 \%, P<0.0001)$, and current smokers $(88.38 \%$ vs. $7.70 \%, P<0.0001)$ among men with LLM compared to those without LLM. Furthermore, prevalence of urinary hesitancy $(16.50 \%$ vs. $5.92 \%, P<0.0001)$, daytime LUTS (35.44\% vs. $24.09 \%, P=0.0073)$, and clinical LUTS $(27.66 \%$ vs. $11.00 \%, P<0.0001)$ was significantly higher in men with LLM compared to those without LLM. The prevalence of incomplete urine emptying, urinary frequency, and nocturia was also higher in participants with LLM compared to those without LLM, but these differences were not statistically significant (all $P>0.05$; Table 1).

\section{Association between LUTS and other clinicopathological characteristics of NHANES study subjects}

We then compared the demographic characteristics and other covariates between men with or without LUTS such as urinary hesitancy, incomplete emptying, urinary frequency and nocturia (Supplementary Table 1). The prevalence of LLM and ALLM was significantly higher among men with LUTS compared to those without LUTS. The prevalence of hypertension $(50.66 \%$ vs. $37.67 \%, P=0.0321)$, current or former smokers $(P=$ $0.0251)$, LLM $(28.79 \%$ vs. $11.40 \%, P<0.0001)$, and $\operatorname{ALLM}(25.18 \%$ vs. $3.37 \%, P<0.0001)$ was significantly higher in study subjects with urinary hesitancy compared to those without urinary hesitancy. The prevalence of ALLM was significant higher in participants with incomplete urine emptying compared to those without incomplete urine emptying $(16.93 \%$ vs. $3.58 \%, P<$ $0.0001)$. The prevalence of LLM was not statistically significant between subjects with or without incomplete urine emptying (18.17\% vs. $12.04 \%, P=0.0827)$. Moreover, men with urinary frequency and nocturia showed higher prevalence of LLM and ALLM compared to those without urinary frequency and nocturia, but the differences were not statistically significant (all $P>$ $0.05)$.

We further compared demographics of participants with or without daytime LUTS and clinical LUTS. The prevalence of hypertension $(54.25 \%$ vs. $33.25 \%$, $P<0.0001)$, diabetes (18.44\% vs. $10.78 \%, P=0.0019)$, sleep disorder $(14.36 \%$ vs. $8.17 \%, P=0.0006)$, current or former smokers $(P=0.0472)$, higher comorbidity index $(P<0.0001)$, LLM $(17.58 \%$ vs. $10.98 \%, P=$ $0.0073)$ and ALLM (8.64\% vs. $3.69 \%, P=0.0020)$ was significantly higher in men with daytime LUTS than those without daytime LUTS (Supplementary Table 2). Furthermore, prevalence of hypertension $(57.91 \%$ vs. $35.70 \%, P<0.0001)$, diabetes $(22.15 \%$ vs. $11.31 \%, P=$ $0.0019)$, current or former smokers $(P=0.0010)$, higher comorbidity conditions $(P<0.0001)$, LLM $(26.72 \%$ vs. $10.55 \%, P<0.0001)$ and ALLM $(16.82 \%$ vs. $3.16 \%, P$ $<0.0001)$ was significantly higher among men with clinical LUTS compared to those without clinical LUTS (Supplementary Table 3).

\section{Association between LLM and LUTS}

We performed weighted multiple regression analysis to determine the association between LLM and ALLM with LUTS. The estimated odd ratios (ORs) for the relationship between LLM and LUTS are shown in Table 2 and those for the association between ALLM and LUTS are shown in Table 3.

In the non-adjusted model, urinary hesitancy $(\mathrm{OR}=3.04$, 95\% CI: 1.77-5.24, $P<0.0001$ ), daytime LUTS (OR = 1.56, 95\% CI: $1.07-2.29, P=0.0211)$ and clinical LUTS $(\mathrm{OR}=2.35,95 \% \mathrm{CI}: 1.52-3.63, P=0.0001)$ were significantly associated with LLM. However, the association between LLM and LUTS parameters such as incomplete urine emptying, urinary frequency, and nocturia was not statistically significant (all $P>0.05$ ). In the adjusted model, LLM was associated with significantly higher risk of urinary hesitancy $(\mathrm{OR}=7.76$, 95\% CI: $3.58-16.82, P<0.0001)$, incomplete urine emptying (OR $=2.49,95 \% \mathrm{CI}: 1.28-4.85, P=0.0070)$, urinary frequency $(\mathrm{OR}=3.28,95 \% \mathrm{CI}: 1.81-5.93, P<$ $0.0001)$, daytime LUTS (OR $=3.88,95 \%$ CI: $2.37=6.35$, $P<0.0001)$ and clinical LUTS (OR $=8.11,95 \%$ CI: 4.26$15.46, P<0.0001$ ), but was not associated with nocturia $(\mathrm{OR}=1.40,95 \%$ CI: $0.86-2.29, P=0.1755)$.

Furthermore, adjusted model results showed that ALLM was significantly associated with urinary hesitancy (OR $=17.97,95 \%$ CI: $6.88-46.90, P<0.0001)$, incomplete urine emptying $(\mathrm{OR}=4.68,95 \% \mathrm{CI}: 2.04-10.70, P=$ $0.0003)$, daytime LUTS (OR $=2.47,95 \%$ CI: $1.20-5.08$, $P=0.0136)$, and clinical LUTS $(\mathrm{OR}=12.18,95 \% \mathrm{CI}$ : 5.26-28.22, $P<0.0001$ ), but was not associated with urinary frequency and nocturia (all $P>0.05$ ).

In addition, we observed significant differences in results from the non-adjusted and adjusted models for the association between LLM and LUTS parameters such as incomplete urine emptying (non-adjusted, $\mathrm{OR}=1.34,95 \%$ CI: 0.78-2.32; adjusted, OR $=2.49,95 \%$ CI: $1.28-4.85$ ), urinary frequency (non-adjusted, OR $=1.35,95 \% \mathrm{CI}$ : $0.85-2.16$; adjusted, $\mathrm{OR}=3.28,95 \% \mathrm{CI}: 1.81-5.93$ ) for LLM, and the association between ALLM and LUTS (non-adjusted, OR $=1.56,95 \%$ CI: 0.87-2.77; adjusted, $\mathrm{OR}=2.47,95 \%$ CI: 1.20-5.08). These differences may be due to confounding bias between lean mass loss and LUTS in the non-adjusted model, but they were eliminated in the adjusted model. Therefore, we postulate that adjusted OR is of greater clinical significance. 
Table 2. Association between low lean mass and lower urinary tract symptoms, weighted.

\begin{tabular}{|c|c|c|}
\hline & \multicolumn{2}{|c|}{ OR $(95 \%$ CI $), P$ value } \\
\hline & Non-adjusted $(n=959)$ & Adjusted $(n=877)$ \\
\hline \multicolumn{3}{|c|}{ Urinary hesitancy } \\
\hline No & Reference & Reference \\
\hline Yes & $3.04(1.77,5.24)<0.0001$ & $7.76(3.58,16.82)<0.0001$ \\
\hline \multicolumn{3}{|c|}{ Incomplete emptying } \\
\hline No & Reference & Reference \\
\hline Yes & $1.34(0.78,2.32) 0.2892$ & $2.49(1.28,4.85) 0.0070$ \\
\hline \multicolumn{3}{|c|}{ Urinary frequency } \\
\hline No & Reference & Reference \\
\hline Yes & $1.35(0.85,2.16) 0.2042$ & $3.28(1.81,5.93)<0.0001$ \\
\hline \multicolumn{3}{|c|}{ Nocturia } \\
\hline No & Reference & Reference \\
\hline Yes & $1.10(0.74,1.63) 0.6363$ & $1.40(0.86,2.29) 0.1755$ \\
\hline \multicolumn{3}{|c|}{ Daytime LUTS } \\
\hline No & Reference & Reference \\
\hline Yes & $1.56(1.07,2.29) 0.0211$ & $3.88(2.37,6.35)<0.0001$ \\
\hline \multicolumn{3}{|c|}{ Clinical LUTS } \\
\hline No & Reference & Reference \\
\hline Yes & $2.35(1.52,3.63) 0.0001$ & $8.11(4.26,15.46)<0.0001$ \\
\hline
\end{tabular}

Abbreviations: LUTS: lower urinary tract symptoms; OR: odd ratio.

Adjusted for age, BMI, race, education level, income, insurance, alcohol intake, diabetes, hypertension, smoking status, comorbidity index.

Table 3. Association between alternate low lean mass and lower urinary tract symptoms, weighted.

\begin{tabular}{|c|c|c|}
\hline & \multicolumn{2}{|c|}{ OR $(95 \%$ CI $), P$ value } \\
\hline & Non-adjusted $(n=959)$ & Adjusted $(n=877)$ \\
\hline \multicolumn{3}{|c|}{ Urinary hesitancy } \\
\hline No & Reference & Reference \\
\hline Yes & $6.18(3.21,11.93)<0.0001$ & $17.97(6.88,46.90)<0.0001$ \\
\hline \multicolumn{3}{|c|}{ Incomplete emptying } \\
\hline No & Reference & Reference \\
\hline Yes & $3.47(1.81,6.63) 0.0002$ & $4.68(2.04,10.70) 0.0003$ \\
\hline \multicolumn{3}{|c|}{ Urinary frequency } \\
\hline No & Reference & Reference \\
\hline Yes & $1.34(0.66,2.73) 0.4166$ & $1.70(0.69,4.21) 0.2486$ \\
\hline \multicolumn{3}{|c|}{ Nocturia } \\
\hline No & Reference & Reference \\
\hline Yes & $1.10(0.60,2.01) 0.7492$ & $1.16(0.54,2.51) 0.6975$ \\
\hline \multicolumn{3}{|c|}{ Daytime LUTS } \\
\hline No & Reference & Reference \\
\hline Yes & $1.56(0.87,2.77) 0.1335$ & $2.47(1.20,5.08) 0.0136$ \\
\hline \multicolumn{3}{|c|}{ Clinical LUTS } \\
\hline No & Reference & Reference \\
\hline Yes & $3.73(2.06,6.74)<0.0001$ & $12.18(5.26,28.22)<0.0001$ \\
\hline
\end{tabular}

Abbreviations: LUTS: lower urinary tract symptoms; OR: odd ratio.

Adjusted for age, BMI, race, education level, income, insurance, alcohol intake, diabetes, hypertension, smoking status, comorbidity index.

\section{DISCUSSION}

In this cross-sectional study included 959 men aged $\geq 40$ years old, we investigated the relationship between LLM and LUTS using weighted multiple regression analysis based on the NHANES dataset (2005-2006). After adjusting for covariates such as age, BMI, race, education level, income, insurance, alcohol intake, diabetes, hypertension, smoking status and comorbidity index, the risk of urinary hesitancy, incomplete urine emptying, urinary frequency, daytime LUTS, and clinical LUTS was significantly higher among men with 
LLM compared to those without LLM. Moreover, ALLM was associated with urinary hesitancy, incomplete urine emptying, daytime LUTS and clinical LUTS. Since both LLM and ALLM showed positive association with LUTS, we postulate that treatment and management of lean mass loss at a younger age might be beneficial to improve or alleviate LUTS.

To the best of our knowledge, this is the first study to evaluate the association between LLM and LUTS in older US men. Previous studies have reported association of LUTS with several other clinicopathological factors. Gacci et al. reported that central obesity was a risk factor for LUTS progression after prostatic surgery to resolve benign prostatic enlargement or prostate cancer [19]. A cross-sectional study by Soma et al. including 710 community-dwelling Japanese participants aged $\geq 60$ years old demonstrated that subjects with LUTS were potentially frailer than those without LUTS, indicating a positive correlation between frailty and LUTS [15]. Huang et al. reported that urinary incontinence was independently associated with frailty among older adults living in Taiwanese rural communities [17]. Bauer et al. also observed that frailty was common among older men with LUTS and should be considered during the initial urological evaluation [16]. Both obesity and frailty were associated with LLM [14, 18], thereby suggesting a possible relationship between LLM and LUTS. Our results demonstrated that men with LLM or ALLM were associated with a higher risk for LUTS than men without LLM. Pacini et al. investigated the association between LUTS and clinicopathological parameters in systemic sclerosis patients, and demonstrated a significant positive correlation between overactive bladder and sarcopenia, which was consistent with our findings [20].

LLM and LUTS share several common risk factors. Many studies have shown that both LLM and LUTS are highly prevalent in aged individuals [2, 8, 21]. Moreover, a cross-sectional study by Fantus et al. reported that sleep disorder was associated with the occurrence of nocturia and daytime LUTS [22]. Furthermore, accumulated evidences have also proven that reductions in the duration and quality of sleep and increases in the prevalence of circadian rhythm and sleep disorder with age enhanced the risk of LLM by promoting proteolysis, modifying the body composition, and increasing the risk of insulin resistance [23]. These findings demonstrated that sleep disorders may also act as a common risk factor of LLM and LUTS. Thus, we designated sleep disorder as a covariate in the adjusted weighted multiple regression models to obtain more reliable results.

The exact mechanisms underlying the association between LLM and LUTS are not clear. Both LLM and
LUTS are closely related to hormonal deregulation. Hypogonadism is commonly observed in subjects with LLM, persistent hypogonadism promotes prostate enlargement and worsens LUTS through a hypogonadism-obesity-benign prostatic hyperplasiaLUTS model [24, 25]. In our study, the prevalence of obesity was significantly higher in men with LUTS compared to non-obese individuals $(68.54 \%$ vs. $34.11 \%, P<0.0001$ ), which was consistent with previous studies. Moreover, lean mass loss was associated with deficiencies in growth hormone $(\mathrm{GH})$ and testosterone [9, 26]. Patients with adult growth hormone deficiency (AGHD) showed reduced lean body mass and muscle strength compared to than healthy individuals [27]. In addition, AGHD patients who received GH treatment showed a lower prevalence of LLM compared to those that did not receive GH treatment [28]. Several studies also have demonstrated that declining testosterone levels are associated with LUTS. Kim et al. reported that testosterone levels were significantly reduced in patients with severe nocturia [29]. Rabijewski et al. studied prevalence of erectile dysfunction and LUTS in middle-aged and elderly men with pre-diabetes, and reported a positive association between lower testosterone levels with severe LUTS [30]. Wu et al. reported higher prevalence of nocturia in men with reduced testosterone levels who underwent trans-urethral prostate resection as well [31]. Decreased testosterone levels may contribute to LUTS by inducing hyperactivity of the autonomic nervous system. Testosterone could play a critical role in the reflex activity of the pelvic autonomic nervous system by interacting with the postsynaptic non-genomic receptors located in the epithelium of the urethra and bladder, thereby increasing bladder volume and compliance, and by reducing detrusor pressure at male maximum flow to inhibit detrusor activity $[32,33]$. Both testosterone and $\mathrm{GH}$ regulated the skeletal muscle protein synthesis and degradation through several common signaling pathways [34]. GH enhances the metabolic effects of testosterone, which in turn affects the neuroendocrine rhythm of GH [35]. The secretion of these two hormones is positively related and interplayed closely. Hence, we postulate a relationship between LLM and LUTS. However, further studies are necessary to understand the potential mechanisms regulating this association between LLM and LUTS.

Our study has several strengths. Firstly, our study was based on the data from NHANES, which was a nationwide, population-based sampling data obtained using a standard protocol for NHANES project. Moreover, the study was more representative because the analysis was performed by considering an appropriate NHANES sampling weight and involved a complex 
multistage cluster survey design. Secondly, we adjusted for confounding covariates to ensure our results reliable and could be applied to a wider range of individuals. We also selected covariates mainly based on previous studies in order to assess the relationship of LUTS with other exposure variables. However, there are several limitations in this study. Due to the cross-sectional study design, we could not obtain the casual relationship between LLM and LUTS. Besides, LUTS was based on self-reported symptoms by the study subjects. This may have resulted in recall bias and might impact the accuracy of our results. Moreover, this study was based on only one NHANES cycle (2005 to 2006) because only this cycle included data regarding both on appendicular lean mass and urinary symptoms. Therefore, the sample capacity of our study was relatively small. Moreover, the data of some potential confounders such as the use of diuretics, alpha blockers, surgery for benign prostate hyperplasia and some other medications, was not available in the NHANES data. We cannot completely rule out residual confounders due to unmeasured or unknown covariates. Therefore, the influence of these confounders on the association between LLM and LUTS is not known. Finally, the data regarding muscle strength was not available in NHANES to assess LLM.

In conclusion, our study demonstrated that both LLM and ALLM were associated with higher risk of LUTS in men aged $\geq 40$ years old. Therefore, we postulate that management and treatment of LLM and ALLM at a younger age might be beneficial to alleviate LUTS. However, further studies are still needed to validate our findings.

\section{METHODS}

\section{Data source}

We obtained publicly available data from the National Health and Nutrition Examination Survey (NHANES), a cross-sectional study to assess the nutrition and health status of adults and children in the United States [36]. NHANES is conducted on a repeated two-year cycle by the National Center for Health Statistics (NCHS). All NHANES data are publicly available at https://www.cdc.gov/nchs/nhanes/.

Our study was based on the data from NHANES 2005 to 2006, since only this cycle contained information on both appendicular lean mass and questionnaires related to urinary symptoms. The NCHS Ethics Review Board granted the approval for NHANES to conduct human subject study. Written informed consent was obtained from all each participant.

\section{Study population}

Men aged $\geq 40$ years who both answered questionnaires on LUTS and underwent body composition measurements were enrolled in our study.

Out of the originally enrolled 10348 individuals in the NHANES 2005-2006, we excluded females $(n=5268)$, individuals aged below 40 years old $(n=3537)$, individuals missing the examination data relating to LLM $(n=507)$, individuals missing LUTS questionnaires data $(n$ =63) and participants with prostate cancer $(n=14)$. Therefore, our final analysis included 959 men aged $\geq 40$ years (Figure 1).

\section{Body composition measurements}

Body composition measurements were conducted by well-trained technicians using dual energy X-ray absorptiometry (DXA) QDR-4500 Hologic Scanner (Bedford, MA). DXA scans were not performed for participants with a self-reported use of radiographic contrast material (barium) in the seven days before scans, weight above 450 pounds and height above $192.5 \mathrm{~cm}$. The data on total lean mass, appendicular lean mass (ALM), bone mineral content, bone area and fat mass were reported and available in NHANES 20052006. Although total lean mass was available, we could not find a proper cut-off value to define low lean mass according to previous studies regarding total lean mass data. Therefore, we used ALM to define LLM and ALLM in our analysis. ALM was defined as the sum of muscle mass of all four upper and lower extremity limbs. In our analysis, we define ALM adjusted for BMI $\left(\mathrm{ALM}_{\mathrm{BMI}}\right)$ and ALM to classify low lean mass according to FNIH in 2014 [37]. For men, low lean mass (LLM) was defined as $\mathrm{ALM}_{\mathrm{BMI}}<0.789$ and alternate low lean mass (ALLM) was defined as ALM $<19.75 \mathrm{~kg}$. Low lean mass and alternate low lean mass were used as exposure variables.

\section{Lower urinary tract symptoms}

LUTS were assessed based on answers to the following four questions, including: (1)"Do you usually have trouble starting to urinate?" for urinary hesitancy; (2)“After urinating, does your bladder feel empty?" for incomplete urine emptying; (3) "How often do you have urinary leakage?" for urinary frequency; and (4) "During the past 30 days, how many times per night did you most typically get up to urinate, from the time you went to bed at night until the time you got up in the morning?" for nocturia.

Nocturia was defined as waking up at least twice per night to urinate during sleep. If a participant answered 
yes to urinary hesitancy, or incomplete emptying, or at least 1 to urinary frequency, he was considered to have a daytime LUTS. An individual with 2 or more the mentioned symptoms above (urinary hesitancy, incomplete emptying, urinary frequency and nocturia) was considered to have clinical LUTS. Urinary hesitancy, incomplete emptying, urinary frequency, nocturia, daytime LUTS and clinical LUTS were used as outcome variables.

\section{Covariates}

The selection of covariates was mainly based on previous studies that assessed the relationship of LUTS with other exposure variables $[22,38]$. We included data regarding demographic (age, race), socioeconomic conditions (education level, ratio of family income to poverty, insurance), lifestyle (alcohol intake, smoking status) and health status (body mass index, sleep disorder, diabetes, other comorbidities) as covariates.

Comorbidity index reflected the health status of patients and was of great significance for disease management, clinical treatment and prognosis [39]. Comorbid conditions included diabetes mellitus, congestive heart failure, chronic obstructive pulmonary disease (emphysema and/or chronic bronchitis), coronary artery disease, cancer and hypertension. The data about comorbid conditions was obtained from answers to the questionnaire in NHANES by the study subjects. An ordinal comorbidity index was then estimated based on the total number of combined condition reported by the study subjects as reported previously [22]. Hypertension was defined based on self-reported hypertension diagnosis, or a blood pressure measurement $\geq 140 / 90$ $\mathrm{mmHg}$ [40]. Diabetes was defined as self-reported diabetes diagnosis, use of oral hypoglycemic agents or insulin, a fasting glucose level $\geq 126 \mathrm{mg} / \mathrm{dL}$, or a plasma glucose level $\geq 200 \mathrm{mg} / \mathrm{dL}$ at $2 \mathrm{~h}$ after oral glucose tolerance test [41]. Congestive heart failure, chronic obstructive pulmonary disease, coronary artery disease and cancer were all based on a self-reported answer regarding previous diagnosis.

Categorical variables included race (Mexican American, other Hispanic, non-Hispanic White, nonHispanic Black and other races), education level (less than high school, high school or general educational development and above high school), ratio of family income to poverty $(\leq 1$ and $>1)$, whether have insurance (yes or not), alcohol intake (never, up to once a week, 2-3 times a week, 4-6 times a week and daily or more), body mass index (BMI in $\mathrm{Kg} / \mathrm{m}^{2}$; categorized as $<25$ for normal weight, 25-29.9 as overweight and $\geq 30$ as obese), diabetes (yes or not), smoking status (never, former or current). All detailed measurement process of these variable was publicly available at https://www.cdc.gov/nchs/nhanes/.

\section{Statistical analysis}

Statistical analysis was conducted according to CDC guidelines using Empower software (https://www.empowerstats.com/; X\&Y solutions, Inc., Boston MA) and $\mathrm{R}$ version 3.4 .3 (https://www.Rproject.org, The $\mathrm{R}$ Foundation) [42]. All statistical analysis was performed by considering appropriate NHANES sampling weights using the complex multistage cluster survey design. Continuous variables were represented as mean with standard deviation, whereas, categorical variables were presented as frequency or percentage. Weighted student's $t$ test (for continuous variables) or weighted chi-square test (for categorical variables) were used to assess the differences between groups classified according to LLM and LUTS status. Weighted multiple regression analysis was performed to estimate the independent relationship between exposure variables (LLM, ALLM) and outcomes variables (urinary hesitancy, incomplete emptying, urinary frequency, nocturia, daytime LUTS, clinical LUTS) using non-adjusted model and model adjusted for age, BMI, race, education level, income, insurance, alcohol intake, diabetes, hypertension, smoking status and comorbidity index. $P<0.05$ was considered statistically significant.

\section{AUTHOR CONTRIBUTIONS}

Zheng Qin: Data analysis, Software, Writing-Original draft; Junjie Zhao: Formal analysis, Writing-Original draft; Jiameng Li: Software; Qinbo Yang: Methodology, Software; Jiwen Geng: Data analysis; Ruoxi Liao: Methodology; Baihai Su: Conceptualization, Funding acquisition, Writing-Reviewing and Editing. All the authors approved the final version.

\section{ACKNOWLEDGMENTS}

We thank the volunteers for their participation, gratefully acknowledge the staff of the NHANES (the National Health and Nutrition Examination Surveys) for their involvement in this study.

\section{CONFLICTS OF INTEREST}

The authors declare no conflicts of interest related to this study.

\section{FUNDING}

This work was supported by the National Natural Science Foundation of China [Grant No. 82000702], the Science 
and Technology Achievement Transformation Fund of West China Hospital of Sichuan University [Grant No. CGZH19006], the 1.3.5 project for disciplines of excellence from West China Hospital of Sichuan University [Grant No. ZYJC21010] and Med+ Biomaterial Institute of West China Hospital/West China School of Medicine of Sichuan University [Grant No. ZYME20001].

\section{REFERENCES}

1. Gratzke C, Bachmann A, Descazeaud A, Drake MJ, Madersbacher S, Mamoulakis C, Oelke M, Tikkinen KAO, Gravas S. EAU Guidelines on the Assessment of Non-neurogenic Male Lower Urinary Tract Symptoms including Benign Prostatic Obstruction. Eur Urol. 2015; 67:1099-109.

https://doi.org/10.1016/i.eururo.2014.12.038 PMID:25613154

2. Alawamlh OAH, Goueli R, Lee RK. Lower Urinary Tract Symptoms, Benign Prostatic Hyperplasia, and Urinary Retention. Med Clin North Am. 2018; 102:301-11. https://doi.org/10.1016/i.mcna.2017.10.005 PMID:29406059

3. MacKenzie KR, Aning JJ. Managing lower urinary tract symptoms in men. Practitioner. 2016; 260:11-16. PMID:27337754

4. Abrams P. Managing lower urinary tract symptoms in older men. BMJ. 1995; 310:1113-17. https://doi.org/10.1136/bmj.310.6987.1113 PMID: $\underline{7742679}$

5. Bavendam TG, Norton JM, Kirkali Z, Mullins C, Kusek JW, Star RA, Rodgers GP. Advancing a Comprehensive Approach to the Study of Lower Urinary Tract Symptoms. J Urol. 2016; 196:1342-49.

https://doi.org/10.1016/j.juro.2016.05.117 PMID:27341750

6. Welliver C, Feinstein L, Ward JB, Fwu CW, Kirkali Z, Bavendam T, Matlaga BR, McVary KT, and Urologic Diseases in America Project. Trends in Lower Urinary Tract Symptoms Associated with Benign Prostatic Hyperplasia, 2004 to 2013: the Urologic Diseases in America Project. J Urol. 2020; 203:171-76. https://doi.org/10.1097/JU.0000000000000499 PMID:31430232

7. Cooper R, Bann D, Wloch EG, Adams JE, Kuh D. "Skeletal muscle function deficit" in a nationally representative British birth cohort in early old age. J Gerontol A Biol Sci Med Sci. 2015; 70:604-07. https://doi.org/10.1093/gerona/glu214 PMID:25431354

8. Geng J, Deng L, Qiu S, Bian H, Cai B, Li Y, Li J, Qin Z, Yang Q, Dong B, Su B. Low lean mass and cognitive performance: data from the National Health and Nutrition Examination Surveys. Aging Clin Exp Res. 2021. [Epub ahead of print].

https://doi.org/10.1007/s40520-021-01835-w

PMID:33786800

9. Marzetti E, Calvani R, Tosato M, Cesari M, Di Bari M, Cherubini A, Collamati A, D'Angelo $E$, Pahor $M$, Bernabei R, Landi F, and SPRINTT Consortium. Sarcopenia: an overview. Aging Clin Exp Res. 2017; 29:11-17.

https://doi.org/10.1007/s40520-016-0704-5 PMID:28155183

10. Landi F, Calvani R, Cesari M, Tosato M, Martone AM, Ortolani E, Savera G, Salini S, Sisto A, Picca A, Marzetti E. Sarcopenia: An Overview on Current Definitions, Diagnosis and Treatment. Curr Protein Pept Sci. 2018; 19:633-38.

https://doi.org/10.2174/138920371866617060711 $\underline{3459}$

PMID:28595526

11. Papadopoulou SK. Sarcopenia: A Contemporary Health Problem among Older Adult Populations. Nutrients. 2020; 12:1293.

https://doi.org/10.3390/nu12051293

PMID: $\underline{32370051}$

12. Landi F, Liperoti R, Fusco D, Mastropaolo S, Quattrociocchi D, Proia A, Tosato M, Bernabei R, Onder G. Sarcopenia and mortality among older nursing home residents. J Am Med Dir Assoc. 2012; 13:121-26.

https://doi.org/10.1016/j.jamda.2011.07.004 PMID:21856243

13. Yang TK, Chang CC, Chang HC, Yang HJ, Huang KH. Factors Associated with Bothersome Lower Urinary Tract Symptoms in Middle-Aged Men Receiving Health Checkup. Sci Rep. 2019; 9:901.

https://doi.org/10.1038/s41598-018-37605-4

PMID:30696907

14. Choi KM. Sarcopenia and sarcopenic obesity. Korean J Intern Med. 2016; 31:1054-60. https://doi.org/10.3904/kjim.2016.193 PMID:27809450

15. Soma O, Hatakeyama S, Imai A, Matsumoto T, Hamano I, Fujita $\mathrm{N}$, Iwamura $\mathrm{H}$, Okamoto $\mathrm{T}$, Yamamoto $\mathrm{H}$, Tobisawa Y, Yoneyama T, Yoneyama T, Hashimoto Y, et al. Relationship between frailty and lower urinary tract symptoms among community-dwelling adults. Low Urin Tract Symptoms. 2020; 12:128-36. https://doi.org/10.1111/luts.12292 PMID: $\underline{31642610}$

16. Bauer SR, Jin C, Kamal P, Suskind AM. Association Between Lower Urinary Tract Symptoms and Frailty in 
Older Men Presenting for Urologic Care. Urology. 2021; 148:230-34.

https://doi.org/10.1016/j.urology.2020.09.041

PMID:33049232

17. Huang CY, Lee WJ, Lin HP, Chen RC, Lin CH, Peng LN, Chen LK. Epidemiology of frailty and associated factors among older adults living in rural communities in Taiwan. Arch Gerontol Geriatr. 2020; 87:103986.

https://doi.org/10.1016/j.archger.2019.103986 PMID:31901458

18. Fougere B, Sourdet S, Lilamand $M$, Tabue-Teguod $M$, Teysseyre B, Dupuy C, Vellas B, Rolland Y, Nourhashemi $F$, van Kan GA. Untangling the overlap between frailty and low lean mass: Data from Toulouse frailty day hospital. Arch Gerontol Geriatr. 2018; 75:209-13.

https://doi.org/10.1016/i.archger.2017.12.013 PMID:29426485

19. Gacci M, Sebastianelli A, Salvi M, De Nunzio C, Tubaro A, Gravas S, Moncada I, Serni S, Maggi M, Vignozzi L. The Impact of Central Obesity on Storage Luts and Urinary Incontinence After Prostatic Surgery. Curr Urol Rep. 2016; 17:61.

https://doi.org/10.1007/s11934-016-0620-4 PMID:27432378

20. Pacini G, Paolino S, Trombetta AC, Goegan F, Pizzorni C, Alessandri E, Patane M, Gotelli E, Ferrari G, Cattelan F, Ghio M, Casabella A, Smith V, Cutolo M. Lower urinary tract symptoms in systemic sclerosis: a detailed investigation. Rheumatology (Oxford). 2020; 59:1315-24.

https://doi.org/10.1093/rheumatology/kez438 PMID:31586421

21. Foong YC, Chherawala N, Aitken D, Scott D, Winzenberg T, Jones G. Accelerometer-determined physical activity, muscle mass, and leg strength in community-dwelling older adults. J Cachexia Sarcopenia Muscle. 2016; 7:275-83.

https://doi.org/10.1002/icsm.12065

PMID:27239404

22. Fantus RJ, Packiam VT, Wang $\mathrm{CH}$, Erickson BA, Helfand BT. The Relationship between Sleep Disorders and Lower Urinary Tract Symptoms: Results from the NHANES. J Urol. 2018; 200:161-66.

https://doi.org/10.1016/j.juro.2018.01.083

PMID:29408214

23. Piovezan RD, Abucham J, Dos Santos RV, Mello MT, Tufik S, Poyares D. The impact of sleep on age-related sarcopenia: Possible connections and clinical implications. Ageing Res Rev. 2015; 23:210-20.

https://doi.org/10.1016/j.arr.2015.07.003 PMID:26216211

24. Cohen PG. Abdominal obesity and intra-abdominal pressure: a new paradigm for the pathogenesis of the
hypogonadal-obesity-BPH-LUTS connection. Horm Mol Biol Clin Investig. 2012; 11:317-20.

https://doi.org/10.1515/hmbci-2012-0030

PMID:25436690

25. Vandenput L, Mellstrom D, Karlsson MK, Orwoll E, Labrie F, Ljunggren O, Ohlsson C. Serum estradiol is associated with lean mass in elderly Swedish men. Eur J Endocrinol. 2010; 162:737-45. https://doi.org/10.1530/eje-09-0696 PMID:20061331

26. Aversa A, Morgentaler A. The practical management of testosterone deficiency in men. Nat Rev Urol. 2015; 12:641-50.

https://doi.org/10.1038/nrurol.2015.238 PMID:26458755

27. Lissett CA, Shalet SM. Effects of growth hormone on bone and muscle. Growth Horm IGF Res. 2000 (Suppl B); 10:S95-101. https://doi.org/10.1016/s1096-6374(00)80018-0 PMID: 10984262

28. Zheng XY, Cheng QF, Long J, Wang YT, Gong LL, Wei Q, Luo R, Liao K, Ren W. Prevalence of low lean mass in patients with adult growth hormone deficiency with or without low-dose growth hormone therapy. Clin Endocrinol (Oxf). 2019; 90:834-41.

https://doi.org/10.1111/cen.13958

PMID:30817014

29. Kim MK, Zhao C, Kim SD, Kim DG, Park JK. Relationship of sex hormones and nocturia in lower urinary tract symptoms induced by benign prostatic hyperplasia. Aging Male. 2012; 15:90-95.

https://doi.org/10.3109/13685538.2012.659715 PMID:22385128

30. Rabijewski M, Papierska L, Kuczerowski R, Piątkiewicz P. Hormonal determinants of erectile dysfunction and lower urinary tract symptoms in middle-aged and elderly men with prediabetes. Aging Male. 2015; 18:256-64.

https://doi.org/10.3109/13685538.2015.1083972 PMID:26444448

31. Wu Y, Pan H, Wang WM, Xu D, Zhang L, Gu ZQ, Bai Q, Qi J, Huang HF. A possible relationship between serum sex hormones and benign prostatic hyperplasia/lower urinary tract symptoms in men who underwent transurethral prostate resection. Asian J Androl. 2017; 19:230-33.

https://doi.org/10.4103/1008-682X.171575 PMID:26763548

32. Karazindiyanoglu S, Cayan S. The effect of testosterone therapy on lower urinary tract symptoms/bladder and sexual functions in men with symptomatic late-onset hypogonadism. Aging Male. 2008; 11:146-49. 
https://doi.org/10.1080/13685530802290438

PMID:18821291

33. Koritsiadis G, Stravodimos K, Mitropoulos D, Doumanis G, Fokitis I, Koritsiadis S, Constantinides C. Androgens and bladder outlet obstruction: a correlation with pressure-flow variables in a preliminary study. BJU Int. 2008; 101:1542-46.

https://doi.org/10.1111/i.1464-410x.2008.07521.x PMID:18294304

34. Agarwal M, Naghi J, Philip K, Phan A, Willix RD Jr, Schwarz ER. Growth hormone and testosterone in heart failure therapy. Expert Opin Pharmacother. 2010; 11:1835-44. https://doi.org/10.1517/14656566.2010.485615 PMID:20486829

35. Mauras N. Growth hormone and sex steroids. Interactions in puberty. Endocrinol Metab Clin North Am. 2001; 30:529-44. https://doi.org/10.1016/s0889-8529(05)70200-0 PMID:11571929

36. Skubisz C, Kern A. National Health and Nutrition Examination Survey: National Health and Nutrition Examination Survey. 2010.

37. Studenski SA, Peters KW, Alley DE, Cawthon PM, McLean RR, Harris TB, Ferrucci L, Guralnik JM, Fragala MS, Kenny AM, Kiel DP, Kritchevsky SB, Shardell MD, et al. The FNIH Sarcopenia Project: Rationale, Study Description, Conference Recommendations, and Final Estimates. J Gerontol A Biol Sci Med Sci. 2014; 69:54758.

https://doi.org/10.1093/gerona/glu010

PMID:24737557
38. Thurmon KL, Breyer BN, Erickson BA. Association of bowel habits with lower urinary tract symptoms in men: findings from the 2005-2006 and 2007-2008 National Health and Nutrition Examination Survey. J Urol. 2013; 189:1409-14.

https://doi.org/10.1016/j.juro.2012.10.008

PMID:23063806

39. Charlson ME, Charlson RE, Peterson JC, Marinopoulos SS, Briggs WM, Hollenberg JP. The Charlson comorbidity index is adapted to predict costs of chronic disease in primary care patients. J Clin Epidemiol. 2008; 61:1234-40.

https://doi.org/10.1016/i.jclinepi.2008.01.006 PMID: 18619805

40. Fryar CD, Ostchega $Y$, Hales CM, Zhang G, KruszonMoran D. Hypertension Prevalence and Control Among Adults: United States, 2015-2016. NCHS Data Brief. 2017; 289:1-8.

PMID:29155682

41. McClure ST, Schlechter H, Oh S, White K, Wu BW, Pilla SJ, Maruthur NM, Yeh HC, Miller ER, Appel LJ. Dietary intake of adults with and without diabetes: results from NHANES 2013-2016. BMJ Open Diabetes Res Care. 2020; 8:e001681.

https://doi.org/10.1136/bmjdrc-2020-001681

PMID:33099509

42. Johnson $C L$, Paulose-Ram R, Ogden CL, Carroll MD, Kruszon-Moran D, Dohrmann SM, Curtin LR. National health and nutrition examination survey: analytic guidelines, 1999-2010. Vital Health Stat 2. 2013; 161:1-24. PMID:25090154 


\section{SUPPLEMENTARY MATERIALS}

\section{Supplementary Tables}

Supplementary Table 1. Participants demographics by lower urinary tract symptoms, weighted.

\begin{tabular}{|c|c|c|c|c|c|c|c|c|c|c|c|c|c|c|}
\hline & \multirow{2}{*}{$\begin{array}{c}\text { Overall } \\
(n=959) \\
\end{array}$} & \multicolumn{3}{|c|}{ Urinary hesitancy } & \multicolumn{3}{|c|}{ Incomplete emptying } & \multicolumn{3}{|c|}{ Urinary frequency } & \multicolumn{3}{|c|}{ Nocturia } & \\
\hline & & Yes $(n=68)$ & No $(n=891)$ & $P$ value & Yes $(n=97)$ & No $(n=862)$ & $P$ value & Yes $(n=141)$ & No $(n=818)$ & $P$ value & Yes $(n=266)$ & No $(n=593)$ & $P$ value & \\
\hline Age (years) & $52.08 \pm 7.91$ & $51.91 \pm 8.27$ & $52.09 \pm 7.88$ & 0.8553 & $51.61 \pm 8.47$ & $52.13 \pm 7.84$ & 0.5358 & $52.39 \pm 8.23$ & $52.03 \pm 7.86$ & 0.6343 & $52.02 \pm 7.68$ & $52.26 \pm 8.55$ & 0.6808 & \\
\hline \multicolumn{15}{|l|}{ Race $(\%)$} \\
\hline Mexican American & 6.30 & 5.51 & 6.36 & 0.9353 & 4.39 & 6.52 & 0.8920 & 7.30 & 6.14 & 0.4107 & 7.64 & 5.84 & 0.6698 & \\
\hline Other Hispanic & 2.43 & 3.64 & 2.33 & & 1.66 & 2.51 & & 2.54 & 2.41 & & 2.43 & 2.43 & & \\
\hline Non-Hispanic White & 77.08 & 76.47 & 77.13 & & 80.43 & 76.70 & & 73.10 & 77.71 & & 73.72 & 78.23 & & \\
\hline Non-Hispanic Black & 9.98 & 11.19 & 9.89 & & 9.72 & 10.01 & & 14.24 & 9.32 & & 11.51 & 9.47 & & \\
\hline Other Races & 4.21 & 3.19 & 4.28 & & 3.80 & 4.25 & & 2.82 & 4.42 & & 4.70 & 4.04 & & \\
\hline \multicolumn{15}{|l|}{ Education level (\%) } \\
\hline Less than high school & 5.82 & 3.94 & 5.97 & 0.7358 & 7.39 & 5.64 & 0.3024 & 4.64 & 6.00 & 0.7658 & 5.82 & 5.82 & 0.9920 & \\
\hline High school or GED & 35.91 & 38.60 & 35.70 & & 41.50 & 35.27 & & 37.87 & 35.60 & & 35.58 & 36.02 & & \\
\hline Above high school & 58.27 & 57.46 & 58.34 & & 51.11 & 59.09 & & 57.49 & 58.40 & & 58.60 & 58.16 & & \\
\hline \multicolumn{15}{|l|}{ RIP (\%) } \\
\hline$\leq 1$ & 8.30 & 8.05 & 9.99 & 0.4686 & 8.01 & 10.82 & 0.3375 & 8.29 & 8.31 & 0.9950 & 7.60 & 10.35 & 0.1797 & \\
\hline$>1$ & 91.70 & 91.95 & 90.01 & & 91.99 & 89.18 & & 91.71 & 91.69 & & 92.40 & 89.65 & & \\
\hline \multicolumn{15}{|c|}{ Alcohol intake per week (\%) } \\
\hline Never & 20.19 & 18.55 & 20.31 & 0.9849 & 16.83 & 20.56 & 0.3200 & 18.79 & 20.41 & 0.8778 & 17.35 & 21.12 & 0.2336 & \\
\hline Up to once a week & 41.44 & 42.77 & 41.34 & & 37.68 & 41.85 & & 43.59 & 41.10 & & 37.91 & 42.60 & & \\
\hline 2-3 times a week & 16.77 & 16.51 & 16.79 & & 20.54 & 16.36 & & 18.31 & 16.53 & & 19.30 & 15.94 & & \\
\hline 4-6 times a week & 11.45 & 10.39 & 11.53 & & 16.80 & 10.86 & & 11.38 & 11.46 & & 14.18 & 10.56 & & \\
\hline Daily or more & 10.15 & 11.79 & 10.03 & & 8.14 & 10.37 & & 7.93 & 10.49 & & 11.26 & 9.78 & & \\
\hline \multicolumn{15}{|l|}{ BMI (\%) } \\
\hline Normal weight & 20.66 & 22.26 & 20.54 & 0.8933 & 21.79 & 20.53 & 0.9005 & 18.40 & 21.02 & 0.0528 & 21.68 & 20.32 & 0.6666 & \\
\hline Overweight & 40.28 & 37.77 & 40.47 & & 41.19 & 40.17 & & 49.88 & 38.77 & & 41.69 & 39.80 & & \\
\hline Obese & 39.06 & 39.97 & 38.99 & & 37.01 & 39.30 & & 31.71 & 40.22 & & 36.63 & 39.89 & & \\
\hline Insurance (\%) & 83.45 & 84.33 & 83.38 & 0.8366 & 83.44 & 83.45 & 0.9987 & 88.73 & 82.62 & 0.0820 & 86.15 & 82.53 & 0.1902 & \\
\hline Hypertension (\%) & 38.61 & 50.66 & 37.67 & 0.0321 & 42.39 & 38.18 & 0.4163 & 58.42 & 35.51 & $<0.0001$ & 53.09 & 33.70 & $<0.0001$ & \\
\hline Diabetes (\%) & 12.73 & 16.56 & 12.43 & 0.3200 & 15.91 & 12.37 & 0.3177 & 22.14 & 11.26 & 0.0006 & 21.58 & 9.73 & $<0.0001$ & \\
\hline CHF (\%) & 4.69 & 4.15 & 4.73 & 0.8274 & 5.77 & 4.56 & 0.5902 & 10.69 & 3.75 & 0.0005 & 10.55 & 2.69 & $<0.0001$ & \\
\hline COPD (\%) & 4.77 & 4.97 & 4.75 & 0.9351 & 4.44 & 7.64 & 0.1576 & 12.15 & 3.62 & $<0.0001$ & 5.30 & 4.59 & 0.6510 & \\
\hline CAD (\%) & 5.43 & 8.57 & 5.18 & 0.2304 & 5.23 & 7.20 & 0.4115 & 5.50 & 5.42 & 0.9714 & 10.25 & 3.79 & 0.0001 & \\
\hline Cancer (\%) & 3.63 & 7.22 & 3.35 & 0.0961 & 7.41 & 3.20 & 0.0342 & 3.94 & 3.58 & 0.8368 & 5.74 & 2.91 & 0.0415 & \\
\hline Sleep disorder (\%) & 9.74 & 4.29 & 10.16 & 0.2559 & 3.36 & 10.47 & 0.0682 & 16.27 & 8.72 & 0.0227 & 14.36 & 8.17 & 0.0006 & \\
\hline \multicolumn{15}{|l|}{ Smoking status (\%) } \\
\hline Never & 39.71 & 27.69 & 40.65 & 0.0251 & 29.78 & 40.85 & 0.0831 & 36.39 & 40.23 & 0.5821 & 34.20 & 41.58 & 0.1121 & \\
\hline Former & 33.64 & 47.85 & 32.53 & & 36.71 & 33.29 & & 33.52 & 33.66 & & 37.78 & 32.24 & & \\
\hline Current & 26.65 & 24.45 & 26.82 & & 33.51 & 25.86 & & 30.09 & 26.11 & & 28.02 & 26.18 & & \\
\hline \multicolumn{15}{|l|}{ Comorbidity index (\%) } \\
\hline 0 & 50.96 & 40.61 & 51.77 & 0.0833 & 44.24 & 51.72 & 0.2172 & 29.45 & 54.32 & $<0.0001$ & 50.96 & 35.86 & 56.08 & $<0.0001$ \\
\hline 1 & 34.60 & 36.76 & 34.43 & & 35.92 & 34.45 & & 44.61 & 33.03 & & 34.60 & 37.08 & 33.76 & \\
\hline 2 & 9.98 & 18.12 & 9.34 & & 11.86 & 9.76 & & 15.70 & 9.08 & & 9.98 & 18.25 & 7.17 & \\
\hline$\geq 3$ & 4.47 & 4.51 & 4.47 & & 7.98 & 4.07 & & 10.24 & 3.57 & & 4.47 & 8.82 & 2.99 & \\
\hline LLM (\%) & 12.67 & 28.79 & 11.40 & $<0.0001$ & 18.17 & 12.04 & 0.0827 & 17.21 & 11.96 & 0.0941 & 14.10 & 12.18 & 0.4377 & 0.4377 \\
\hline ALLM (\%) & 4.95 & 25.18 & 3.37 & $<0.0001$ & 16.93 & 3.58 & $<0.0001$ & 6.62 & 4.69 & 0.3459 & 6.94 & 4.27 & 0.0980 & 0.0980 \\
\hline
\end{tabular}

Abbreviations: BMI: body mass index; GED: general educational development; RIP: ratio of family income to poverty; CHF: congestive heart failure; COPD: chronic obstructive pulmonary disease; CAD: Coronary artery disease; LLM: low lean mass; ALLM: alternate low lean mass. 
Supplementary Table 2. Participants demographics by daytime LUTS, weighted.

\begin{tabular}{|c|c|c|c|c|}
\hline & Overall & Daytime LUTS & Non-Daytime LUTS & $P$ value \\
\hline & $(n=959)$ & $(n=253)$ & $(n=706)$ & \\
\hline Age (years) & $52.08 \pm 7.91$ & $51.72 \pm 8.14$ & $52.20 \pm 7.83$ & 0.4084 \\
\hline \multicolumn{5}{|l|}{ Race $(\%)$} \\
\hline Mexican American & 6.30 & 6.64 & 6.18 & \multirow[t]{5}{*}{0.7429} \\
\hline Other Hispanic & 2.43 & 3.04 & 2.21 & \\
\hline Non-Hispanic White & 77.08 & 74.61 & 77.93 & \\
\hline Non-Hispanic Black & 9.98 & 11.78 & 9.37 & \\
\hline Other Races & 4.21 & 3.93 & 4.30 & \\
\hline \multicolumn{5}{|l|}{ Education level (\%) } \\
\hline Less than high school & 5.82 & 5.40 & 5.96 & \multirow[t]{3}{*}{0.0962} \\
\hline High school or GED & 35.91 & 41.63 & 33.95 & \\
\hline Above high school & 58.27 & 52.97 & 60.09 & \\
\hline \multicolumn{5}{|l|}{ RIP (\%) } \\
\hline$\leq 1$ & 8.30 & 7.86 & 9.57 & \multirow[t]{2}{*}{0.4026} \\
\hline$>1$ & 91.70 & 92.14 & 90.43 & \\
\hline \multicolumn{5}{|c|}{ Alcohol intake per week (\%) } \\
\hline Never & 20.19 & 20.23 & 20.18 & \multirow[t]{5}{*}{0.8819} \\
\hline Up to once a week & 41.44 & 41.60 & 41.39 & \\
\hline 2-3 times a week & 16.77 & 18.09 & 16.33 & \\
\hline 4-6 times a week & 11.45 & 11.60 & 11.40 & \\
\hline Daily or more & 10.15 & 8.48 & 10.71 & \\
\hline \multicolumn{5}{|l|}{ BMI $(\%)$} \\
\hline Normal weight & 20.66 & 20.76 & 20.63 & \multirow[t]{3}{*}{0.0569} \\
\hline Overweight & 40.28 & 46.10 & 38.27 & \\
\hline Obese & 39.06 & 33.14 & 41.10 & \\
\hline Insurance (\%) & 83.45 & 12.40 & 17.97 & 0.0430 \\
\hline Hypertension (\%) & 38.61 & 54.25 & 33.25 & $<0.0001$ \\
\hline Diabetes (\%) & 12.73 & 18.44 & 10.78 & 0.0019 \\
\hline CHF (\%) & 4.69 & 7.54 & 3.71 & 0.0142 \\
\hline COPD $(\%)$ & 4.77 & 9.96 & 2.99 & $<0.0001$ \\
\hline CAD (\%) & 5.43 & 6.81 & 4.96 & 0.2705 \\
\hline Cancer $(\%)$ & 3.63 & 6.05 & 2.80 & 0.0188 \\
\hline Sleep disorder $(\%)$ & 9.74 & 14.36 & 8.17 & 0.0006 \\
\hline \multicolumn{5}{|l|}{ Smoking state (\%) } \\
\hline Never & 39.71 & 33.38 & 41.88 & \multirow[t]{3}{*}{0.0472} \\
\hline Former & 33.64 & 38.82 & 31.87 & \\
\hline Current & 26.65 & 27.80 & 26.25 & \\
\hline \multicolumn{5}{|l|}{ Comorbidity index (\%) } \\
\hline 0 & 50.96 & 34.28 & 56.67 & \multirow[t]{4}{*}{$<0.0001$} \\
\hline 1 & 34.60 & 42.45 & 31.91 & \\
\hline 2 & 9.98 & 14.98 & 8.26 & \\
\hline$\geq 3$ & 4.47 & 8.30 & 3.16 & \\
\hline LLM $(\%)$ & 12.67 & 17.58 & 10.98 & 0.0073 \\
\hline $\operatorname{ALLM}(\%)$ & 4.95 & 8.64 & 3.69 & 0.0020 \\
\hline
\end{tabular}

Abbreviations: BMI: body mass index; RIP: ratio of family income to poverty; GED: general educational development; CHF: congestive heart failure; COPD: chronic obstructive pulmonary disease; CAD: Coronary artery disease; LLM: low lean mass; ALLM: alternate low lean mass. 
Supplementary Table 3. Participants demographics by clinical LUTS, weighted.

\begin{tabular}{|c|c|c|c|c|}
\hline & Overall & Clinical LUTS & Non-Clinical LUTS & $P$ value \\
\hline & $(n=959)$ & $(n=134)$ & $(n=825)$ & \\
\hline Age (years) & $52.08 \pm 7.91$ & $51.59 \pm 8.28$ & $52.15 \pm 7.85$ & 0.4611 \\
\hline \multicolumn{5}{|l|}{ Race $(\%)$} \\
\hline Mexican American & 6.30 & 5.72 & 6.39 & \multirow[t]{5}{*}{0.3210} \\
\hline Other Hispanic & 2.43 & 3.74 & 2.23 & \\
\hline Non-Hispanic White & 77.08 & 75.17 & 77.37 & \\
\hline Non-Hispanic Black & 9.98 & 13.44 & 9.46 & \\
\hline Other Races & 4.21 & 1.93 & 4.55 & \\
\hline \multicolumn{5}{|l|}{ Education level (\%) } \\
\hline Less than high school & 5.82 & 4.72 & 5.98 & \multirow[t]{3}{*}{0.4817} \\
\hline High school or GED & 35.91 & 40.52 & 35.21 & \\
\hline Above high school & 58.27 & 54.76 & 58.80 & \\
\hline \multicolumn{5}{|l|}{ RIP $(\%)$} \\
\hline$\leq 1$ & 8.30 & 7.89 & 11.02 & \multirow[t]{2}{*}{0.2352} \\
\hline$>1$ & 91.70 & 92.11 & 88.98 & \\
\hline \multicolumn{5}{|c|}{ Alcohol intake per week (\%) } \\
\hline Never & 20.19 & 15.73 & 20.84 & \multirow[t]{5}{*}{0.5387} \\
\hline Up to once a week & 41.44 & 39.47 & 41.73 & \\
\hline 2-3 times a week & 16.77 & 19.94 & 16.31 & \\
\hline 4-6 times a week & 11.45 & 14.12 & 11.06 & \\
\hline Daily or more & 10.15 & 10.74 & 10.06 & \\
\hline \multicolumn{5}{|l|}{ BMI $(\%)$} \\
\hline Normal weight & 20.66 & 18.30 & 21.02 & \multirow[t]{3}{*}{0.7717} \\
\hline Overweight & 40.28 & 40.90 & 40.18 & \\
\hline Obese & 39.06 & 40.80 & 38.80 & \\
\hline Insurance (\%) & 83.45 & 87.06 & 82.90 & 0.2423 \\
\hline Hypertension (\%) & 38.61 & 57.91 & 35.70 & $<0.0001$ \\
\hline Diabetes (\%) & 12.73 & 22.15 & 11.31 & 0.0019 \\
\hline CHF (\%) & 4.69 & 9.65 & 3.94 & 0.0048 \\
\hline COPD $(\%)$ & 4.77 & 8.77 & 4.16 & 0.0238 \\
\hline CAD (\%) & 5.43 & 9.19 & 4.86 & 0.0457 \\
\hline Cancer $(\%)$ & 3.63 & 8.81 & 2.85 & 0.0009 \\
\hline Sleep disorder (\%) & 9.74 & 10.61 & 9.61 & 0.7962 \\
\hline \multicolumn{5}{|l|}{ Smoking state (\%) } \\
\hline Never & 39.71 & 24.59 & 41.99 & \multirow[t]{3}{*}{0.0010} \\
\hline Former & 33.64 & 41.52 & 32.45 & \\
\hline Current & 26.65 & 33.89 & 25.56 & \\
\hline \multicolumn{5}{|l|}{ Comorbidity index (\%) } \\
\hline 0 & 50.96 & 31.29 & 53.92 & \multirow[t]{4}{*}{$<0.0001$} \\
\hline 1 & 34.60 & 40.05 & 33.78 & \\
\hline 2 & 9.98 & 19.08 & 8.60 & \\
\hline$\geq 3$ & 4.47 & 9.59 & 3.70 & \\
\hline LLM $(\%)$ & 12.67 & 26.72 & 10.55 & $<0.0001$ \\
\hline $\operatorname{ALLM}(\%)$ & 4.95 & 16.82 & 3.16 & $<0.0001$ \\
\hline
\end{tabular}

Abbreviations: BMI: body mass index; GED: general educational development; RIP: ratio of family income to poverty; CHF: congestive heart failure; COPD: chronic obstructive pulmonary disease; CAD: Coronary artery disease; LLM: low lean mass; ALLM: alternate low lean mass. 